\section{Ixabepilone shows activity in hormone-refractory prostate cancer}

Taxanes stabilize the microtubules involved in cell division, thereby inhibiting tumor growth. As some tumors are taxane-resistant, alternatives such as the epothilones (which also stabilize microtubules) are being investigated. A group of US researchers has examined the activity of one epothilone, ixabepilone, in 42 patients with chemotherapy-naive, metastatic, hormone-refractory prostate cancer.

Patients were given ixabepilone $40 \mathrm{mg} / \mathrm{m}^{2}$ intravenously once every 21 days, for a minimum of two treatments. Therapy continued until the tumor progressed, unacceptable toxicity occurred, or the patient decided to withdraw. Median follow-up was 25 months. In all, 14/42 patients had a confirmed partial PSA response, defined as a $\geq 50 \%$ reduction in serum PSA level from baseline. The PSA levels of 10 of these patients declined by $>80 \%$, and two patients achieved undetectable PSA levels. No patient achieved a confirmed complete objective response (disappearance of all tumor tissue). Median progression-free survival was estimated at 6 months, and median survival was 18 months. Eleven patients required dose reduction to the prespecified level of $32 \mathrm{mg} / \mathrm{m}^{2}$ and one to the prespecified level of $25 \mathrm{mg} / \mathrm{m}^{2}$, because of low granulocyte or platelet counts.

The authors claim that, compared to their group's previous studies of single agents in hormone-refractory prostate cancer, ixabepilone shows clinically documented antitumor activity. It also compares favorably with results reported for docetaxel plus prednisone. The effects of dose level and dose schedule on ixabepilone activity are, as yet, unknown, so further study is recommended.

Katherine Sole

Original article Hussain et al. (2005) Ixabepilone (epothilone $\mathrm{B}$ analogue BMS-247550) is active in chemotherapy-naive patients with hormone-refractory prostate cancer: a Southwest Oncology Group trial S0111. J Clin Oncol 23: 8724-8792

\section{Endoscopic treatment of vesicoureteral reflux is safe but not highly effective}

The standard treatment for vesicoureteral reflux (VUR) is open surgical ureteric reimplantation, but a less-invasive alternative is endoscopic subureteral injection of a bulking agent. Mevorach et al. have conducted a prospective, multicenter, 2-year, US trial, examining the safety and efficacy of first-line endoscopic treatment with one of the available bulking agents (synthetic calcium hydroxyapatite) in children with VUR and traditional indications for surgery.

Synthetic calcium hydroxyapatite was injected under cystoscopic guidance in 98 patients (155 ureters) with grades II-IV VUR. Of these, 44 received one injection, 51 received two injections, and 3 received three injections. Data were analyzed for the 86 patients who completed the initial follow-up period. Across the 10 centers, $32 \%$ of patients were cured at 1 and 2 years. Ureteral cure rates were $46 \%$ at 1 year and $40 \%$ at 2 years. The primary treatment center, however, produced higher success rates, with a 2 year cure rate of $66 \%$ of patients and $72 \%$ of ureters. These better results were probably the result of the primary center's greater experience. Three patients experienced serious adverse events, none of which were related to the procedure or material used.

Endoscopic subureteral injection with synthetic calcium hydroxyapatite was safe, but not highly effective, when compared to the rates of cure achieved by open surgery (typically 95$98 \%$ in grades I-IV VUR). Before a new technique can replace open surgical repair of VUR, it must demonstrate long-term surgical success and include a comparison to medically treated patients, say the authors.

Katherine Sole

Original article Mevorach RA et al. (2006) Results of a 2-year multicenter trial of endoscopic treatment of vesicoureteral reflux with synthetic calcium hydroxyapatite. J Urol 175: 288-291

\section{BCG plus mitomycin is more effective than BCG alone in bladder cancer}

Most systemic anticancer regimens combine drugs for increased efficacy, but this approach has rarely been applied to intravesicular treatment of bladder cancer. The current standard treatment for early-stage or superficial bladder cancer is resection followed by immunotherapeutic Bacillus Calmette-Guérin (BCG) therapy. Combination therapy with ELECTROMOTIVE ADMINISTRATION of the

\section{GLOSSARY}

ELECTROMOTIVE

ADMINISTRATION

Therapy that uses a local electric current to introduce the ions of a medicine into the tissues 